\title{
Robotic lung cancer surgery: from simple to complex, from surgery to clinical study
}

\author{
Yu Han, Yajie Zhang, Chengqiang Li, Su Yang, Hecheng Li \\ Department of Thoracic Surgery, Ruijin Hospital, Shanghai Jiao Tong University School of Medicine, Shanghai 200025, China \\ Correspondence to: Hecheng Li. Department of Thoracic Surgery, Ruijin Hospital, Shanghai Jiao Tong University School of Medicine, No. 197, Ruijin \\ Er Road, Shanghai 200025, China. Email: lihecheng2000@hotmail.com.
}

Submitted Jul 19, 2019. Accepted for publication Sep 24, 2019.

doi: $10.21037 /$ jtd.2019.09.79

View this article at: http://dx.doi.org/10.21037/jtd.2019.09.79

\section{Introduction}

The robotic surgical system has been increasingly utilized for thoracic surgeries in recent years. For the treatment of lung cancer, the safety and feasibility of robotic lobectomy, segmentectomy, and even sleeve resection have been demonstrated by a series studies (1-3). Robotic-assisted thoracic surgery (RATS) was initiated in May 2015 in our department, and, as of June 2019, more than 1,000 cases have been performed, including lung surgery, esophagectomy for esophageal tumors, and mediastinal tumor resection. For robotic-assisted lung surgery, 530 patients underwent lobectomy, 204 patients underwent segmentectomy, and 8 patients underwent sleeve resection. We started from robotic lobectomy and segmentectomy, and sleeve resection was carried out when we acquired greater proficiency of the robotic surgical technique. To summarize our experience and improve the efficacy of robotic lung cancer surgery, several retrospective studies and prospective clinical trials have been conducted in our department.

\section{Robotic lobectomy}

Based on the Society of Thoracic Surgeons General Thoracic database (STS-GTD), the proportion of robotic lobectomy has increased from $<1 \%$ in 2009 to $18.1 \%$ in 2016 (4). Robotic lobectomy was mainly used in the treatment for clinical stage I/II non-small-cell lung cancer (NSCLC) $(5,6)$. It is noticeable that the safety and surgical efficacy of robotic lung resection for locally advanced NSCLC has also been demonstrated by an international retrospective study recently (7). In the past several years, the techniques and surgical outcomes of robotic lobectomy have been investigated by several studies, with acceptable morbidity and mortality $(3,5)$. As a new minimal invasive technical method, whether RATS is superior to video-assisted thoracic surgery (VATS) for treatment of lung cancer has become an area of intense research.

In our department, the 4-arm approach was used for robotic lobectomy. We performed all types of robotic lobectomy in a short time since the RATS program started. Uniportal VATS (UVATS) was started in January 2015 in our institution. A retrospective study was then conducted to investigate the early outcomes between RATS and UVATS for NSCLC (8). From January 2015 to September 2016, 153 NSCLC patients who were undergoing RATS or UVATS in our department were enrolled in this study, and each group included 69 cases after propensity score match. No significant differences were observed in complications and other postoperative outcomes such as chest tube duration and hospital stay. However, RATS had advantages in reducing blood loss $(\mathrm{P}=0.037)$ and dissecting higher amounts of lymph node stations $(\mathrm{P}=0.014)$ than UVATS for lung cancer. Although propensity score match analysis was used, bias might have still been present due to the study's retrospective nature and limited sample size. To determine whether RATS lobectomy would be as effective as VATS lobectomy in short-term and long-term outcomes, a prospective random clinical trial (RCT) was carried out in our department (NCT03134534). The sample size was 300 patients, and they had surgical indication for lobectomy. This RCT was initiated on June 2017. Until June 2019, 230 participants were enrolled in the two groups, with 117 patients in RATS group and 113 patients in VATS group. The midterm outcomes were satisfactory in both groups. There was no 
significant difference in overall postoperative complications, length of hospital stays and conversion rates between RATS and VATS groups. This trial is ongoing smoothly.

\section{Robotic segmentectomy}

In recent years, with the greater utilization of computer tomography (CT) for lung cancer screening, small pulmonary lesions have been increasingly identified (9). For the treatment of early stage NSCLC, minimally invasive segmentectomy has been widely accepted because more pulmonary functions can be preserved without the compromise of oncologic outcomes $(10,11)$. The technique of robotic segmentectomy is more complex than robotic lobectomy (12). The surgical complexity of segmentectomy can be classified into three categories based on the degree of surgical difficulty: easy, fairly difficult, and difficult (13). We started from an easy procedure, S6 resection, which only has a single intersegmental dissection surface. Gradually, we performed fairly difficult segmentectomy including on S1, S2, S3 and other segments which have multiple dissection surfaces in contact at obtuse angles. Finally, for difficult segmentectomy, we were able to perform procedures on segments like S1a+2 and S2b+3a, which had a deeply located bronchial pulmonary artery or multiple dissection surfaces in contact at acute angles.

With more than 100 robotic segmentectomies completed by a single surgical team led by Hecheng Li, the learning curve was analyzed with the cumulative sum (CUSUM) method (14). The learning process consists of three phases: the initial learning period ( $1^{\text {st }}$ to $21^{\text {st }}$ operation), the consolidation period $\left(22^{\text {nd }}\right.$ to $46^{\text {th }}$ operation), and the experienced period ( $47^{\text {th }}$ to $104^{\text {th }}$ operation). Specially, we demonstrated the safety and efficacy of robotic combined anatomic subsegmentectomy (CAS) for the first time (15). In this study, 16 patients with cT1N0M0 lesions underwent robotic CAS, a challenging technique. The robotic surgical system offers great benefits with accuracy and flexibility for this kind of delicate surgical operation. Furthermore, we have conducted prospective clinical trials focusing on the clinical issues of segmentectomy. To explore whether energy instruments or stapling devices were more suitable to dissect intersegmental plans in segmentectomy, a randomized controlled trial (NCT03192904) was carried out in 2017. Seventy patients were recruited and this trial has been completed recently. The results of this clinical trial will be revealed soon. Another ongoing clinical trial, which was initiated in 2018 (NCT03516500), aimed to test the safety and effectiveness of identifying lung intersegmental plane by injecting iron sucrose. Until July 2019, 17 patients were enrolled in this clinical trial and intersegmental plane can be identified successfully in a part of cases using this method. Both clinical trials may offer high quality evidence for the clinical practice of segmentectomy.

\section{Robotic sleeve resection}

Bronchial sleeve resection is a highly technique-demanding procedure. Although robotic lobectomy and segmentectomy have seen widespread use, reports concerning robotic sleeve resection are sparse with limited cases $(2,16,17)$. The largest case series of robotic sleeve lobectomy was reported by Jiao et al. in 2019 and included 67 cases (18). In this retrospective study, a half-continuous suture technique was used, and the postoperative complication rate was $20.9 \%$, with no deaths occurring within 90 days after surgery.

In our department, robotic sleeve resection was carried out cautiously after we successfully performed more than 150 anatomic lobectomies and segmentectomies. Bronchial anastomosis was performed by continuous running suture combined with interrupted sutures, as we reported in a retrospective study (16). As of June 2019, 8 robotic sleeve resections have been performed at our institution with satisfactory results. However, more high quality studies with larger case numbers are needed to confirm the surgical outcomes of robotic sleeve resection.

\section{Uniportal robotic surgery}

Robotic surgical system possesses several advantages, including a magnified three-dimensional view, manipulator wrist with improved dexterity and tremor filtration system. However, 4-5 incisions are necessary for robotic thoracic surgery at present. On the contrast, uniportal VATS surgery only need one incision, but the techniques of which are complex. The emergence of Uniportal robotic system is a new evolution for minimally invasive thoracic surgery, which combined the superiorities of robotic surgery and uniportal surgery. Recently, Gonzalez-Rivas et al. (19) reported the early experience of subcostal uniportal robotic-assisted lobectomy based on cadavers, showed a bright future for clinical application.

\section{Summary}

In our experience, the surgical technique for robotic 
lung surgery could be improved gradually from simple to complex surgery. Moreover, thinking beyond technique and summarizing the experience of different operations are important undertakings which can improve the level of clinical treatment. In the future, high quality prospective clinical trials are still needed to demonstrate the efficacy of robotic lung cancer surgery. What's more, uniportal robotic thoracic surgery may have bright prospects in clinical practice.

\section{Acknowledgments}

None.

\section{Footnote}

Conflicts of Interest: The authors have no conflicts of interest to declare.

Ethical Statement: The authors are accountable for all aspects of the work in ensuring that questions related to the accuracy or integrity of any part of the work are appropriately investigated and resolved.

\section{References}

1. Liang H, Liang W, Zhao L, et al. Robotic Versus Videoassisted Lobectomy/Segmentectomy for Lung Cancer: A Meta-analysis. Ann Surg 2018;268:254-59.

2. Nakamura H, Taniguchi $Y$, Miwa K, et al. A successful case of robotic bronchoplastic lobectomy for lung cancer. Ann Thorac Cardiovasc Surg 2013;19:478-80.

3. Veronesi G. Robotic lobectomy and segmentectomy for lung cancer: results and operating technique. J Thorac Dis 2015;7:S122-30.

4. Feczko AF, Wang H, Nishimura K, et al. Proficiency of Robotic Lobectomy Based on Prior Surgical Technique in The Society of Thoracic Surgeons General Thoracic Database. Ann Thorac Surg 2019;108:1013-20.

5. Rajaram R, Mohanty S, Bentrem DJ, et al. Nationwide Assessment of Robotic Lobectomy for Non-Small Cell Lung Cancer. Ann Thorac Surg 2017;103:1092-100.

6. Schuchert MJ, Pettiford BL, Pennathur A, et al. Anatomic segmentectomy for stage I non-small-cell lung cancer: comparison of video-assisted thoracic surgery versus open approach. J Thorac Cardiovasc Surg 2009;138:1318-25.e1.

7. Veronesi G, Park B, Cerfolio R, et al. Robotic resection of Stage III lung cancer: an international retrospective study.
Eur J Cardiothorac Surg 2018;54:912-9.

8. Yang S, Guo W, Chen X, et al. Early outcomes of robotic versus uniportal video-assisted thoracic surgery for lung cancer: a propensity score-matched study. Eur J Cardiothorac Surg 2018;53:348-52.

9. Humphrey LL, Deffebach M, Pappas M, et al. Screening for lung cancer with low-dose computed tomography: a systematic review to update the US Preventive services task force recommendation. Ann Intern Med 2013;159:411-20.

10. Yang CF, D'Amico TA. Thoracoscopic segmentectomy for lung cancer. Ann Thorac Surg 2012;94:668-81.

11. Nguyen D, Gharagozloo F, Tempesta B, et al. Long-term results of robotic anatomical segmentectomy for earlystage non-small-cell lung cancer. Eur J Cardiothorac Surg 2019;55:427-33.

12. Cerfolio RJ, Watson C, Minnich DJ, et al. One Hundred Planned Robotic Segmentectomies: Early Results, Technical Details, and Preferred Port Placement. Ann Thorac Surg 2016;101:1089-95; Discussion 1095-6.

13. Oizumi H, Kanauchi N, Kato H, et al. Anatomic thoracoscopic pulmonary segmentectomy under 3-dimensional multidetector computed tomography simulation: a report of 52 consecutive cases. J Thorac Cardiovasc Surg 2011;141:678-82.

14. Zhang Y, Liu S, Han Y, et al. Robotic Anatomical Segmentectomy: An Analysis of the Learning Curve. Ann Thorac Surg 2019;107:1515-22.

15. Li C, Han Y, Han D, et al. Robotic Approach to Combined Anatomic Pulmonary Subsegmentectomy: Technical Aspects and Early Results. Ann Thorac Surg 2019;107:1480-6.

16. Li C, Zhou B, Han Y, et al. Robotic sleeve resection for pulmonary disease. World J Surg Oncol 2018;16:74.

17. Pan X, Gu C, Wang R, et al. Initial Experience of Robotic Sleeve Resection for Lung Cancer Patients. Ann Thorac Surg 2016;102:1892-7.

18. Jiao W, Zhao Y, Qiu T, et al. Robotic Bronchial Sleeve Lobectomy for Central Lung Tumors: Technique and Outcome. Ann Thorac Surg 2019;108:211-8.

19. Gonzalez-Rivas D, Ismail M. Subxiphoid or subcostal uniportal robotic-assisted surgery: early experimental experience. J Thorac Dis 2019;11:231-9.

Cite this article as: Han Y, Zhang Y, Li C, Yang S, Li H. Robotic lung cancer surgery: from simple to complex, from surgery to clinical study. J Thorac Dis 2020;12(2):51-53. doi: 10.21037/jtd.2019.09.79 